\title{
Masculinidades e sofrimento mental: do cuidado singular ao enfrentamento do machismo?
}

\author{
Masculinities and mental distress: \\ from personal care to fight against male sexism?
}

Rafael Pereira Silva (https://orcid.org/0000-0001-5043-8631) ${ }^{1}$

Eduardo Alves Melo (https://orcid.org/0000-0001-5881-4849) ${ }^{2}$

${ }^{1}$ Secretaria Municipal de Saúde do Rio de Janeiro.

R. Afonso Cavalcanti 455,

Cidade Nova. 20211-110

Rio de Janeiro RJ Brasil.

rafaelpereirasilvam@

gmail.com

${ }^{2}$ Escola Nacional de Saúde

Pública Sergio Arouca,

Fundação Oswaldo Cruz.

Rio de Janeiro RJ Brasil.

\begin{abstract}
Mental health problems have great international health relevance. From a multifactorial nature, the health conditions considered here as suffering are influenced by social elements such as the construction of masculinity, notwithstanding the evident increasing criticisms and struggles against male sexism. Given this setting, the paper addresses male mental distress and its care, based on a literature review, according to BVS research, and considering the 2010-2020 period. Twenty-two papers were selected from the research. The results of the study were organized into these categories: Characteristics/Particularities of men's mental distress; Access/Way of seeking help by men in distress and approach/Care of men in mental distress. We can conclude that there is a need for more visibility for the relationship between masculinity and suffering and their care specificities, considering the existence of an apparent silent crisis, the right of men (as people) to care, and the possible contribution, albeit indirect and modest, of addressing men's distress to the fight against machismo.
\end{abstract}

Key words Men, Masculinity, Mental Health
Resumo Os problemas de saúde mental têm grande relevância sanitária internacional. De natureza multifatorial, tais condições de saúde, aqui consideradas como sofrimentos, são influenciadas, inclusive, por elementos sociais, como a construção da masculinidade, em que pese as críticas e lutas cada vez mais evidentes contra o machismo. Diante deste cenário, este artigo aborda o sofrimento mental masculino e seu cuidado, a partir de uma revisão da literatura, tendo como base a BVS e considerando o período de 2010 a 2020. Foram selecionados 22 artigos. Os resultados do estudo foram organizados em torno das categorias: $\mathrm{Ca}$ racterísticas/Particularidades do sofrimento mental de homens; Acesso/Modo de procura por ajuda de homens em sofrimento e Abordagem/Cuidado de homens em sofrimento mental. Conclui-se haver necessidade de mais visibilidade para a relação entre masculinidade e sofrimento mental e suas especificidades no âmbito do cuidado, considerando a existência de uma aparente crise silenciosa, $o$ direito dos homens (enquanto pessoas) ao cuidado bem como a possível contribuição, ainda que indireta e modesta, da abordagem do sofrimento dos homens para a luta contra o machismo.

Palavras-chave Homens, Masculinidade, Saúde Mental 


\section{Introdução}

$\mathrm{Na}$ atualidade, pautas singulares, por vezes denominadas identitárias, tais como as lutas feministas/antimachismo, movimentos antirracistas e contra a LGBTfobia, têm se destacado na arena pública, acompanhando-se, todavia, tanto de críticas sobre possíveis limites ligados ao centramento em seus focos específicos quanto de reações conservadoras do status quo. Mas também de formulações que buscam compreender e conectar elementos constituintes de verdadeiras redes de opressão e desigualdade, como a noção de interseccionalidade, entrecruzando gênero, raça e classe 1 . Neste cenário, e considerando por ora a problemática das relações de gênero, não é demais lembrar os efeitos profundamente nefastos do machismo, tendo nas violências simbólicas e concretas algumas das suas principais expressões. Não é à toa que, em plena pandemia da COVID-19, recaem sobre parte das mulheres, por exemplo, o aumento do risco de sofrer violência domiciliar.

Este artigo, no que pode parecer um movimento paradoxal, busca abordar outra face desta problemática, qual seja, o "lado" dos homens, particularmente seus sofrimentos mentais. Embora o machismo tenha nas mulheres seu principal alvo, entendemos que os homens também são, em alguma medida, por ele afetados. Isso porque a internalização de modelos hegemônicos de masculinidade pode estar ligada tanto à produção de sofrimentos, como também a particularidades (muitas vezes compreendidas como barreiras) na expressão e no reconhecimento de tais sofrimentos.

Há que se destacar que o reconhecimento da influência da masculinidade nos processos de adoecimento, apesar de relativamente recente, não se deu na contemporaneidade. Já na década de 1970, época considerada como marco inicial dos estudos sobre homens e saúde, embora com noções pouco elaboradas e tangenciadas pelas teorias feministas, já se reconhecia tal influência ${ }^{2}$. Nas décadas seguintes, foi possível observar um crescimento dos estudos e discussões acerca das particularidades da saúde de homens e, nos anos 2000, surgiram publicações da OMS que destacavam a importância da implementação de políticas de saúde objetivando atender às necessidades dessa população ${ }^{3}$. No âmbito nacional, a aprovação da Política Nacional de Atenção Integral à Saúde do Homem ${ }^{4}$, apesar de ter representado um importante passo, recebe críticas devido ao aparente centramento em questões urológicas ${ }^{5}$.
Neste trabalho pretendemos explorar a complexa relação entre masculinidade e sofrimento mental; porém, antes de seguirmos nessa exploração, parece-nos necessário explicitar que, adotando o entendimento da obra de Gomes, compreendemos a construção do padrão hegemônico de masculinidade a partir de uma perspectiva relacional de gênero, em que padrões sociais esperados de homens e mulheres surgem com objetivo de diferenciá-los, se tornando normatizações que tendem a ser estereotipadas e internalizadas pelas pessoas ${ }^{6}$.

Por sua vez, os sofrimentos mentais no mundo contemporâneo estão ligados a diversos fatores, incluindo aqueles referidos às implicações do neoliberalismo sobre modos de vida instados a se fazerem tendo como referência a empresa, forjando-se como supostos empreendedores de si mesmos, impelidos a buscar auto realizações frequentemente instáveis, num movimento individualizante e de dissolução de laços sociais ${ }^{7}$, ao que se soma, sobretudo em países com marcada desigualdade social, a precariedade material e dificuldade de manter condições básicas de vida.

Quando se observam dados referentes à saúde mental, identifica-se uma prevalência maior de Transtornos Mentais Comuns (TMC) em mulheres ${ }^{8,9}$. Entretanto, aponta-se para uma maior chance de falsos-negativo entre homens quando se realiza testes de screening em saúde mental ${ }^{10}$. Além disso, observa-se que homens são substancialmente mais susceptíveis ao suicídio do que mulheres e, considerando que transtornos mentais comuns são usualmente associados ao suicídio, acredita-se que exista subdiagnóstico dessas condições na população masculina ${ }^{11}$. Esse reconhecimento deficitário pode ocorrer pois homens parecem ter tendência a associar doença à fraqueza e têm maior dificuldade de expressar as ansiedades e os sentimentos de tristeza do que as mulheres ${ }^{10}$. Por isso, podem ser mais resistentes a relatar sintomas emocionais a entrevistadores. Além disso, especula-se que ainda quando o sofrimento é manifestado, singularidades nos modos de sofrer de homens podem representar uma barreira para o reconhecimento por parte de profissionais de Saúde ${ }^{10}$.

Diante deste quadro, esta revisão buscou caracterizar o sofrimento mental de homens e explorar elementos de seu cuidado.

\section{Metodologia}

Optou-se por realizar uma revisão integrativa da literatura que busca captar, reconhecer e sinteti- 
zar a produção do conhecimento sobre determinado assunto ou tema ${ }^{12}$. A pesquisa foi realizada em junho de 2020 e abarcou trabalhos publicados nos últimos 10 anos (de 2010 a 2020) na Biblioteca Virtual em Saúde (BVS) Brasil por se tratar de uma base com alto grau de indexação de periódicos da área de saúde do Brasil e do mundo. As questões norteadoras que guiaram esta revisão foram: "Quais as particularidades do sofrimento mental do homem?" e "Como se dão as abordagens profissionais do sofrimento mental do homem?". Utilizou-se como descritores: men; masculinity; psychological distress; mental health e stress, psychological. Estes foram dispostos com as seguintes combinações: (men OR masculinity) AND ("mental health"; OR "psychological distress" OR “stress, psychological”). Os critérios de inclusão estabelecidos foram: abordagem da saúde mental do homem (perspectiva de gênero) e idioma (inglês, português e espanhol). Os critérios de exclusão foram: indisponibilidade de acesso ao artigo; abordagem no contexto de uma doença específica (excetuando transtornos mentais); abordagem de subgrupos específicos de homens (por exemplo: homens que trabalham em cenários específicos e recortes por faixa -etária como idosos ou adolescentes). A partir da leitura dos artigos incluídos foi possível definir três categorias de abordagem do tema: Características/Particularidades do sofrimento mental de homens; Acesso/Modo de procura por ajuda de homens em sofrimento e Abordagem/Cuidado de homens em sofrimento mental. Em torno delas foram abordados os principais achados e problematizações do estudo, tendo as relações de gênero como pano de fundo.

\section{Resultados e discussão}

$\mathrm{Na}$ primeira fase da pesquisa foram encontrados 345 trabalhos. Realizou-se primeiramente a leitura dos títulos dos artigos e, com base nos critérios de inclusão e exclusão previamente citados, foram excluídos 306 trabalhos. Em seguida, após leitura dos resumos, foram excluídos 10 trabalhos (em sua maioria por abordarem subgrupos específicos). Por fim, após a leitura na íntegra dos trabalhos, foram incluídos 22 artigos (Figura 1). O Quadro 1 consolida os artigos incluídos, seus respectivos autores, ano e local de publicação em relação às categorias temáticas previamente descritas. Dentre os trabalhos que compõem os resultados desta revisão, 8 são revisões da literatura, 7 são estudos observacionais, 4 são análises de dados secundários, 2 são ensaios e 1 é uma carta aberta. Dos estudos observacionais, 6 utilizaram metodologias qualitativas e apenas 1 utilizou metodologia quantitativa. Desses 7 trabalhos, 6 tiveram a coleta de dados feita com base em entrevista individual e 1 através de grupo focal. Destaca-se que nenhum dos trabalhos incluídos foi realizado no Brasil. O Quadro 2 mostra a disposição dos artigos segundo a categoria temática e país de publicação.

\section{Características/Particularidades do sofrimento mental de homens}

A partir dos artigos incluídos na pesquisa observou-se que, embora se perceba certa confluência nos achados dos autores no que diz respeito à maior prevalência de condições como o abuso de substância e transtornos ligados à impulsividade em homens, há questionamentos no que diz respeito à menor prevalência de transtornos mentais comuns ${ }^{11,13}$. Affleck et al. ${ }^{13}$ destacam a discrepância entre a prevalência de depressão em homens - que é significativamente menor que mulheres - e a incidência de suicídio - substancialmente maior nessa população - como um possível indicativo do subdiagnóstico do transtorno depressivo. Isso porque, segundo apontado pelos autores, a maior parte dos casos de suicídio está associado à depressão. Além disso, os autores apontam que homens são menos propensos a reconhecer sintomas relacionados ao humor devido à não conformidade com noções dominantes de masculinidade e parecem ter uma tendência a reagir de modo distinto ao de mulheres quando enfrentam estresse psicológico. Os autores defendem que os homens parecem engajar-se em comportamentos como o abuso de álcool, a tomada exagerada de risco e a violência devido a um fenômeno descrito na psicanálise como "acting-out". Tal reação além de potencialmente danosa, parece contribuir para que o sofrimento mental não seja reconhecido por profissionais de saúde ${ }^{11}$. Sendo assim, segundo os achados desta revisão, a discrepância entre dados de suicídio e depressão em homens, as possíveis limitações no reconhecimento do sofrimento psicológico e os comportamentos de encenação parecem levar ao que alguns autores têm apontado como a "crise silenciosa" da saúde mental de homens ${ }^{13}$.

No contexto nacional, um estudo observacional realizado na cidade de Pelotas (não incluído nos resultados desta pesquisa devido ao ano de publicação) demonstrou que pelo menos um transtorno psiquiátrico categorizado como me- 


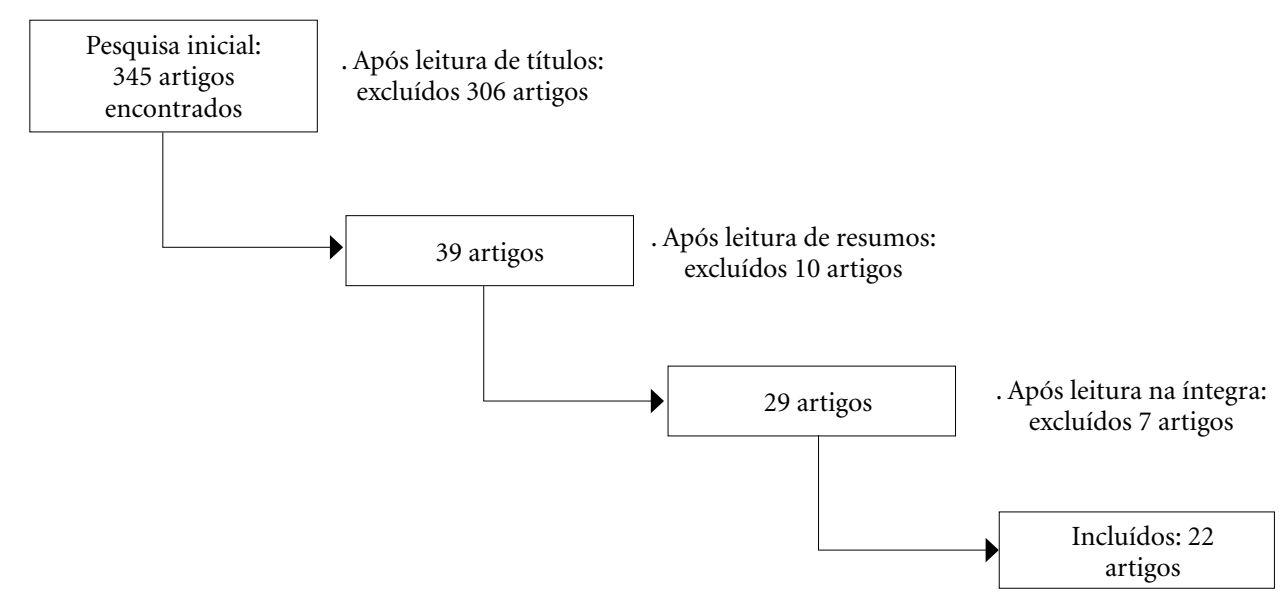

Figura 1. Pesquisa bibliográfica realizada com os descritores men; masculinity; psychological distress; mental health e stress, psychological na BVS Brasil, com seleção por título, idioma (português, inglês e espanhol) e ano de publicação (2010 a 2020).

Fonte: Elaborado pelos autores.

nor esteve presente em $26,5 \%$ das mulheres e em $17,5 \%$ dos homens ${ }^{14}$. Além disso, há também autores brasileiros que afirmam que padrões dominantes de gênero podem levar homens a silenciar questões de saúde ${ }^{15}$ (o que vai ao encontro da ideia da "crise silenciosa") e também levar mulheres a falarem e se queixarem mais de questões ligadas às emoções. Contudo, há aqueles que defendem que as diferenças de prevalência não são necessariamente produto de subdiagnóstico na população masculina. Ludermir et al. ${ }^{16}$, por exemplo, apontam que tais diferenças podem decorrer de desigualdades de gênero, as quais podem produzir impacto negativo na promoção de saúde mental de mulheres, que tendem a sofrer com a dupla jornada e a ainda presente desvalorização no mercado de trabalho. Nós consideramos que o machismo incide sobre a sociedade como um todo, o que leva a evidentes efeitos deletérios na vida de mulheres e que também parece afetar negativamente a saúde mental de homens. Portanto, entendemos que o reconhecimento da maior prevalência de transtornos mentais em mulheres não exclui a possibilidade de existência do sofrimento não reconhecido em homens.

Embora homens pareçam sofrer "em silêncio", há evidências de que não sofrem sozinhos. A partir da análise de dados da 2002 National Survey on Drug Use and Health (pesquisa realizada nos EUA), constatou-se que homens que cometeram violência doméstica reportaram necessidades de saúde mental não tratada duas vezes mais do que aqueles que não cometeram ${ }^{17}$. Além disso,
Fleming et al. ${ }^{18}$ mostraram, a partir de entrevistas com homens do México e dos EUA, que sintomas depressivos estavam associados a atitudes misóginas. Embora devido à natureza exploratória do estudo não se possa definir assertivamente a relação temporal e de causalidade entre sintomas depressivos e atitudes misóginas, os autores deste estudo acreditam que um melhor entendimento dessa relação pode levar a desfechos positivos tanto em homens quanto em mulheres.

Há que se destacar que não se pretende desresponsabilizar os homens perpetradores de violência doméstica ao descrever a possível relação com o sofrimento mental, mas sim discutir um potencial (e relevante) impacto positivo da abordagem de saúde mental de homens que transcende o alívio do sofrimento em si.

Apesar de ter-se percebido uma tendência entre os autores de relacionar padrões hegemônicos de masculinidade a efeitos negativos sobre a saúde mental de homens ${ }^{15,16,19,20}$, observou-se também uma tendência que pode ser entendida como de relativização ou até de oposição a essa visão $0^{21-25}$. Destaca-se o trabalho realizado por Wong et al. ${ }^{22}$ que, a partir de uma perspectiva multidimensional da construção da masculinidade, identificaram que a conformidade com determinadas normatizações em subgrupos específicos de homens pode estar relacionada tanto positiva quanto negativamente com estresse psicológico - há que se dizer que o subgrupo denominado como "tomadores de risco desapegados" (do inglês "detached risk-takers") que está, de forma geral, em confor- 
Quadro 1. Artigos, autores, ano e local de publicação em relação às categorias temáticas.

\begin{tabular}{|c|c|c|c|c|}
\hline Artigos incluídos na revisão & $\begin{array}{l}\text { Autores } \\
\text { Ano/Local }\end{array}$ & $\begin{array}{c}\text { Características } \\
\text { do sofrimento } \\
\text { mental de } \\
\text { homens }\end{array}$ & $\begin{array}{l}\text { Acesso/ } \\
\text { Modo de } \\
\text { procura } \\
\text { por ajuda }\end{array}$ & $\begin{array}{c}\text { Cuidado de } \\
\text { homens em } \\
\text { sofrimento } \\
\text { mental }\end{array}$ \\
\hline $\begin{array}{l}\text { Why it's time to focus on masculinity in } \\
\text { mental health training and clinical practice }\end{array}$ & $\begin{array}{l}\text { Seidler et al. }{ }^{30} \\
\text { 2019/Austrália }\end{array}$ & & & X \\
\hline $\begin{array}{l}\text { Developing a theory-driven framework for } \\
\text { a football intervention for men with severe, } \\
\text { moderate or enduring mental health problems }\end{array}$ & $\begin{array}{l}\text { Such et al. }{ }^{37} \\
\text { 2019/Reino Unido }\end{array}$ & & & $\mathrm{X}$ \\
\hline $\begin{array}{l}\text { Men's Mental Health: Social Determinants } \\
\text { and Implications for Services. }\end{array}$ & $\begin{array}{l}\text { Affleck et al. }{ }^{13} \\
\text { 2018/Canadá }\end{array}$ & $\mathrm{X}$ & & \\
\hline Critical Issues in Men’s Mental Health. & $\begin{array}{l}\text { Bilsker et al. }{ }^{11} \\
\text { 2018/Canadá }\end{array}$ & $\mathrm{X}$ & & \\
\hline $\begin{array}{l}\text { Successful mental health promotion with } \\
\text { men: the evidence from 'tacit knowledge' }\end{array}$ & $\begin{array}{l}\text { Robertson et al. }{ }^{35} \\
2018 /\end{array}$ & & & $\mathrm{X}$ \\
\hline $\begin{array}{l}\text { Are men's misogynistic attitudes associated } \\
\text { with poor mental health and substance use } \\
\text { behaviors? }\end{array}$ & $\begin{array}{l}\text { Fleming et al. }{ }^{18} \\
\text { 2018/México }\end{array}$ & $\mathrm{X}$ & & \\
\hline $\begin{array}{l}\text { How do we improve men's mental health via } \\
\text { primary care? }\end{array}$ & $\begin{array}{l}\text { Cheshire et al. }{ }^{34} \\
\text { 2016/Reino Unido }\end{array}$ & & & $\mathrm{X}$ \\
\hline $\begin{array}{l}\text { Mental health and wellbeing: focus on men's } \\
\text { health }\end{array}$ & \begin{tabular}{|l|} 
Patrick e \\
Robertson \\
2016/Reino Unido \\
\end{tabular} & & & $\mathrm{X}$ \\
\hline $\begin{array}{l}\text { Professional care seeking for mental health } \\
\text { problems among women and men in Europe }\end{array}$ & \begin{tabular}{|l|} 
Buffel et al. ${ }^{26}$ \\
2014/União Europeia
\end{tabular} & $\mathrm{X}$ & & \\
\hline $\begin{array}{l}\text { Men's Mental Health: Beyond Victim- } \\
\text { Blaming }\end{array}$ & \begin{tabular}{|l|} 
Whitley \\
2018/Canadá
\end{tabular} & & & $\mathrm{X}$ \\
\hline $\begin{array}{l}\text { A latent class regression analysis of men's } \\
\text { conformity to masculine norms and } \\
\text { psychological distress }\end{array}$ & $\begin{array}{l}\text { Wong et al. }{ }^{22} \\
\text { 2012/EUA }\end{array}$ & $\mathrm{X}$ & & \\
\hline $\begin{array}{l}\text { Triple jeopardy: impact of partner violence } \\
\text { perpetration, mental health and substance } \\
\text { use on perceived unmet need for mental } \\
\text { health care among men }\end{array}$ & $\begin{array}{l}\text { Lipsky et al. }{ }^{17} \\
\text { 2010/EUA }\end{array}$ & $\mathrm{X}$ & & \\
\hline $\begin{array}{l}\text { Men's mental health: Spaces and places that } \\
\text { work for men }\end{array}$ & $\begin{array}{l}\text { Ogrodniczuk et al }{ }^{36} \\
\text { 2016/Canadá }\end{array}$ & & $\mathrm{X}$ & \\
\hline $\begin{array}{l}\text { Men's Work-Related Stress and Mental } \\
\text { Health }\end{array}$ & $\begin{array}{l}\text { Boettcher et al. }{ }^{19} \\
\text { 2019/EUA }\end{array}$ & $\mathrm{X}$ & & \\
\hline $\begin{array}{l}\text { The Influence of Masculine Norms and } \\
\text { Mental Health on Health Literacy Among Men }\end{array}$ & $\begin{array}{l}\text { Milner et al. }^{20} \\
\text { 2019/EUA }\end{array}$ & $\mathrm{X}$ & & \\
\hline $\begin{array}{l}\text { Masculinity, Social Connectedness, and } \\
\text { Mental Health }\end{array}$ & $\begin{array}{l}\text { McKenzie et al. }{ }^{23} \\
\text { 2018/EUA }\end{array}$ & $\mathrm{X}$ & & \\
\hline $\begin{array}{l}\text { Negotiating Gender Norms to Support Men } \\
\text { in Psychological Distress. }\end{array}$ & $\begin{array}{l}\text { Keohane e } \\
\text { Richardson }{ }^{24} \\
\text { 2018/EUA } \\
\end{array}$ & & $\mathrm{X}$ & \\
\hline $\begin{array}{l}\text { Men's Mental Health Help-Seeking } \\
\text { Behaviors: }\end{array}$ & $\begin{array}{l}\text { Parent et al. }{ }^{27} \\
\text { 2018/EUA }\end{array}$ & & $\mathrm{X}$ & \\
\hline $\begin{array}{l}\text { Problematizing Men's Suicide, Mental } \\
\text { Health, and Well-Being. }\end{array}$ & $\begin{array}{l}\text { Roy et al. }{ }^{32} \\
\text { 2018/Canadá }\end{array}$ & & & $\mathrm{X}$ \\
\hline $\begin{array}{l}\text { Men's Mental Health Promotion } \\
\text { Intervention }\end{array}$ & $\begin{array}{l}\text { Seaton et al. }{ }^{39} \\
\text { 2017/EUA }\end{array}$ & & & $\mathrm{X}$ \\
\hline Men's mental health: a call to social workers & $\begin{array}{l}\text { Shafer e Wendt }{ }^{25} \\
\text { 2015/EUA }\end{array}$ & $\mathrm{X}$ & & \\
\hline
\end{tabular}

Fonte: Elaborado pelos autores. 
Quadro 2. Disposição dos artigos segundo a categoria temática e país de publicação.

\begin{tabular}{|l|c|c|c|c|c|c|}
\hline \multirow{2}{*}{ Categoria temática } & \multicolumn{5}{c|}{ Local de publicação } \\
\cline { 2 - 7 } & EUA & RU & Canadá & Austrália & México & $\begin{array}{c}\text { União } \\
\text { Europeia }\end{array}$ \\
\hline $\begin{array}{l}\text { Características/Particularidades do } \\
\text { sofrimento mental de homens }\end{array}$ & 5 & & 2 & & 1 & 1 \\
\hline $\begin{array}{l}\text { Acesso/Modo de procura por ajuda de } \\
\text { homens em sofrimento }\end{array}$ & 3 & & 1 & & & \\
\hline $\begin{array}{l}\text { Abordagem/Cuidado de homens em } \\
\text { sofrimento mental }\end{array}$ & 2 & 4 & 2 & 1 & & \\
\hline
\end{tabular}

Fonte: Elaborado pelos autores.

midade com normas mais sexistas e patriarcais, apresentou maior sofrimento psicológico. Ainda no sentido de se perceber as construções como multidimensionais, McKenzie et al. ${ }^{23}$ estudaram o estabelecimento de conexões sociais por homens e a mobilização de redes de suporte. Os autores identificaram padrões diversos e, embora alguns entrevistados tenham demonstrado formas de relacionamento mais instrumentais e menos emocionalmente colaborativas, outros apresentaram padrões de conexão social positivos para a saúde emocional. Nesse sentido, destaca-se o alerta feito por Shafer e Wendt ${ }^{25}$ para que as atitudes tradicionalmente percebidas como masculinas não sejam automaticamente percebidas como negativas já que, na perspectiva dos autores, algumas dessas características como a de serem "orientados à ação", e "focados em objetivos" poderiam ser úteis no tratamento. Ainda nessa direção, Whitley ${ }^{21}$ destaca um fenômeno descrito como "culpabilização da vítima” (do Inglês “victim-blaming”), em que se culpabiliza homens por agir em conformidade com padrões de masculinidade, atribuindo-se o sofrimento a esse comportamento e desconsiderando-se a complexidade do fenômeno que envolve o contexto social e determinantes de saúde. Essa visão é entendida como simplista e reducionista e transcendê-la parece ser uma necessidade quando se pensa no cuidado de homens em sofrimento mental ${ }^{24}$.

\section{Acesso/Modo de procura por ajuda de homens em sofrimento}

Apesar de se reconhecer a existência de interseções entre questões referentes às particularidades do sofrimento mental do homem e o modo de busca por ajuda, alguns dos estudos incluídos nesta revisão abordam de forma direta o acesso ou o modo de procura por ajuda de homens em sofrimento. Destaca-se o estudo conduzido por Keohane e Richardson ${ }^{24}$ que, a partir da realização de grupos focais cujos participantes eram homens com maior risco para suicídio ou que exerciam o papel de "gatekeepers" da comunidade, fizeram observações relevantes no que diz respeito à procura, ao recebimento e ao fornecimento de ajuda. Os pesquisadores observaram que os homens consideravam invariavelmente o efeito que as questões psicológicas podem ter sobre a masculinidade. Percebeu-se que normas culturais como conter emoções e manter a "firmeza" frente à adversidade estavam muito presentes entre os participantes. Reconhecer ou admitir o problema foi visto como o primeiro passo mais difícil. Observou-se, ainda, que a busca por ajuda depende de boas conexões feitas no ambiente familiar, no trabalho e nos esportes e que, embora os homens se reconheçam também no papel de “ajudantes”, eles demonstraram receio em não ter a capacidade necessária para a ajuda.

Há que se destacar que, para além do fato de homens em geral buscarem auxílio para questões mentais menos do que mulheres ${ }^{26,27}$, há subgrupos de homens que tendem a acessar o auxílio menos do que outros. Parent et al. ${ }^{27}$ observaram, a partir da aplicação de questionários em uma amostra de homens americanos, que a procura por ajuda (definida no estudo como contato com profissional de saúde mental ou de serviço social nos últimos 12 meses) foi maior entre homens brancos, homossexuais, solteiros, idosos e com depressão mais proeminente.

Autores de um estudo realizado em diversos países da Europa apontaram que fatores socioeconômicos parecem exercer influência na procura por serviços de saúde. Porém, segundo os autores, embora os papéis sociais de homens e mulheres exerçam influência no acesso, esse impacto é variável de acordo com o país estudado ${ }^{26}$. 
Como já mencionado, não foram encontrados estudos realizados no Brasil nesta revisão. Porém, há que se destacar trabalhos que, embora não tenham explorado de forma específica as formas de busca por cuidado por parte de homens no contexto do sofrimento mental, exploraram a temática da busca por cuidado de forma mais ampla, permitindo, em alguma medida, o diálogo com os achados desta revisão. Cunha et al. ${ }^{28}$, por exemplo, mostraram que os homens estudados consideravam a prevenção e os cuidados do corpo como as principais formas de se cuidar; porém, reconhecem que o hábito do autocuidado ainda não foi incorporado à cultura masculino. Figueiredo ${ }^{29}$, por outro lado, destaca que embora de fato exista a ideia de que a baixa presença de homens em serviços de cuidado primário seja associada à desvalorização do autocuidado por essa população, os homens tendem a preferir usar outros serviços como, por exemplo, pronto-socorros ou farmácias. Locais onde, segundo o autor, as demandas são respondidas de forma mais objetiva ${ }^{29}$.

Logo, no contexto nacional, para além das possíveis questões ligadas à identidade masculina, há também aquelas ligadas à organização dos serviços de saúde. Há que se reconhecer, então, que a mudança política e cultural (com a desconstrução dos ideais de masculinidade hegemônicos) não deve ser observada de forma passiva por aqueles que trabalham no setor da saúde. Ao invés disso, esses profissionais talvez devam incorporar os valores dessa mudança em suas práticas e na própria organização do serviço, atentando para o que eventualmente se configura como barreira para os homens e buscando criar estratégias que as contornem, ampliando mecanismos de acolhimento sem, no entanto, serem mera adequação à lógica dos homens.

\section{Abordagem/Cuidado de homens em sofrimento mental}

A partir da leitura dos trabalhos que abordaram as formas de cuidado de homens em sofrimento mental, foi possível perceber que a consideração das particularidades e a adaptação da abordagem de profissionais faz-se relevante $\mathrm{e}^{30-32}$ Seidler et al. ${ }^{30}$, por exemplo, afirmam que quanto mais "gênero-competente" é um profissional de saúde, melhor ela ou ele pode responder aos desafios psicológicos e clínicos de homens. Isso porque a diversidade e a complexidade das expressões da masculinidade parecem se relacionar com a saúde na medida em que pode influenciar a forma de busca por ajuda e o engajamento com o tratamento ${ }^{30}$. Por isso, para os autores, a masculinidade deveria fazer parte do currículo de "saúde mental". Nesse sentido, Patrick e Robertson $^{33}$ destacam a importância de enfermeiras(os) reconhecerem as particularidades na expressão do sofrimento mental de homens e proverem intervenções sensíveis ao gênero. Na mesma direção, Cheshire et al. ${ }^{34}$ descrevem o projeto Atlas, um programa piloto que teve como um dos objetivos tornar a abordagem de médicos generalistas mais sensível ao gênero. Robertson et al..$^{35}$ mostraram que quanto mais sensível à questão de gênero, mais respeitosa(o), e menos julgadora(o), melhor é o efeito de um facilitador na expressão de emoções e na comunicação de homens.

Para além da adaptação de profissionais de saúde, observou-se em alguns trabalhos menção à adaptação do próprio serviço ou ambiente de abordagem ${ }^{18,32}$. Robertson et al. ${ }^{35}$ mostraram que criar um ambiente seguro, onde homens podem se sentir relaxados e confortáveis parece ser importante. Os autores destacam que a maior parte dos serviços é frequentado predominantemente por mulheres se tornando pouco atraente para homens $^{35}$. De forma semelhante, Ogrodniczuk et al. ${ }^{36}$ descreveram iniciativas de transformação de ambientes para que fossem mais acolhedores e receptivos a homens com depressão e ideação suicida. Tais achados vão ao encontro de conclusões de autores de trabalhos realizados no Brasil, como o de Figueiredo ${ }^{29}$, já mencionado anteriormente, que destaca a feminilização dos serviços de APS nacionais.

Parece-nos relevante, contudo, mencionar que o fato de serviços de saúde serem percebidos como "feminilizados" não desloca a responsabilidade da baixa procura de homens unicamente para esses serviços. Na realidade, compreende-se que a resistência desses homens é, também, um reflexo dos constructos sociais hegemônicos de masculinidade, os quais tornam a presença em ambientes percebidos como "feminilizados" desconfortável.

No que diz respeito a estratégias de abordagem, observou-se em dois dos trabalhos incluídos menção à importância de normalizar as experiências de sofrimento de homens ${ }^{33,37}$. Encontrou-se ainda, menção a abordagens "gênero transformativas", em que se objetiva reforçar aspectos positivos da masculinidade ${ }^{32,38}$. Por fim, no que diz respeito a formas de abordagem, observou-se que em 7 dos 14 estudos incluídos em uma revisão sistemática o exercício físico foi utilizado como estratégia para promoção de saúde mental em ho- 
mens $^{39}$. Na mesma direção, Such et al. ${ }^{37}$ mostraram que a intervenção por meio do exercício físico pode ter efeitos terapêuticos na saúde mental de homens na medida em que fomenta a conexão social, a segurança de identidade, a normalização de experiências e a afetividade ${ }^{37}$.

Há que se apontar, contudo, que a própria natureza multifatorial do sofrimento humano aqui compreendido como a desestabilização do conjunto de elementos ou de mundos (passado, presente, familiar, secreto... $)^{40}$ - torna improvável a resposta plena a partir de abordagens pontuais como, por exemplo, a prática de atividade física ou aquelas passíveis de serem realizadas a nível de cuidado individual. Neste sentido, parece-nos oportuno reiterar que o machismo pode se colocar, para parte dos homens, como uma das causas de sofrimento (ao terem que ser provedores ou fortes, por exemplo), mas também como obstáculo ao enfrentamento dos mesmos (sem espaço para mediações e reflexividade). Por fim, destacase que experiências e manifestações de sofrimento não são homogêneas, e que diferenças sociais podem contribuir para conformar configurações distintas de sofrimento e, assim, as formas de enfrentamento podem variar a depender da realidade e do sujeito.

Apesar de ser um risco, a abordagem do sofrimento mental masculino não está fadada a ter, como intenção ou como efeito, a vitimização ou absolvição dos homens. Entendemos que não se trata disso, mas de lembrar que pessoas merecem ter acesso a cuidado diante de sofrimentos e que o sofrimento e o cuidado, apesar de não se estruturarem necessariamente em torno do machismo, podem ser uma via de acesso a processos subjetivos e de produção de "diálogos" que favoreçam ainda que indiretamente, na constituição e vivência de outras possibilidades de masculinidade.

\section{Considerações finais}

Este estudo, ainda que utilizando uma única base na pesquisa bibliográfica, indica que padrões hegemônicos de masculinidade parecem fazer parte tanto da complexa gênese do sofrimento de homens como também impactar nas formas de cuidado desse público. Parece-nos razoável supor, ainda, que o possível subdiagnóstico de transtornos mentais comuns em homens apontado em outros países seja, também, uma realidade no Brasil. Isso porque entendemos que os padrões de masculinidade presentes na sociedade brasileira somados à acentuação da desigualdade socioeconômica e aos desmontes de dispositivos de proteção social, se combinam e se potencializam, podendo influenciar na produção de sofrimentos mentais.

O reconhecimento dos efeitos deletérios do machismo nos próprios homens não deve significar uma desresponsabilização daqueles que muitas vezes utilizam da própria estrutura social machista para oprimir e violentar mulheres. Sendo assim, o cuidado de homens em sofrimento mental não deve anular o debate público de combate às iniquidades de gênero e quiçá possa representar uma pequena contribuição, a depender do modo com que seja operado, para a sua difícil e complexa desconstrução. Os elementos trazidos neste artigo indicam a necessidade de que este tema seja alvo de mais pesquisas, com diferentes abordagens e em diferentes cenários. Isso se torna ainda mais relevante no contexto nacional, visto que não foram encontrados artigos publicados em periódicos brasileiros nesta revisão. 


\section{Colaboradores}

RP Silva atuou na concepção e delineamento do estudo, coleta e análise dos dados, redação, revisão final e aprovação do manuscrito. EA Melo contribuiu na concepção do estudo, análise dos dados, redação, revisão final e aprovação do manuscrito.

\section{Referências}

1. Hirata H. Gênero, classe e raça: interseccionalidade e consubstancialidade das relações sociais. Tempo Soc 2014; 26(1):61-73.

2. Courtenay WH. Constructions of masculinity and their influence on men's well-being: a theory of gender and health. Soc Sci Med 2000; 50(10):1385-1401.

3. World Health Organization (WHO). Boys in the picture. Genebra: WHO; 2000;

4. Brasil. Ministério da Saúde (MS). Departamento de Ações Programáticas Estratégicas. Política nacional de atenção integral à saúde do homem. Brasília: MS; 2008.

5. Hemmi APA, Baptista TWF, Rezende M. O processo de construção da Política Nacional de Atenção Integral à Saúde do Homem. Physis 2020; 30(3):e300321.

6. Separavich MA. A saúde do homem em foco. Interface (Botucatu) 2011; 15(38):957-962.

7. Dardot P, Laval C. A nova razão do mundo: ensaio sobre a sociedade neoliberal. São Paulo: Boitempo; 2016.

8. Costa JSD, Menezes AMB, Olinto MTA, Gigante DP, Macedo S, Britto MAP, Fuchs SC. Prevalência de distúrbios psiquiátricos menores na cidade de Pelotas, RS. Rev Bras Epidemiol 2002; 5(2):164-173.

9. McManus S, Meltzer H, Brugha T, Bebbington P, Jenkin R. Adult psychiatric morbidity in England, 2007: results of a household survey. London: National Centre for Social Research; 2009.

10. Mari JJ, Williams P. Misclassification by psychiatric screening questionnaires. J Chron Dis 1986; 39(5):371-377.

11. Bilsker D, Fogarty AS, Wakefield MA. Critical Issues in Men's Mental Health. Can J Psychiatry 2018; 63(9):590-596.

12. Mendes KDS, Silveira RCCP, Galvão CM. Revisão integrativa: método de pesquisa para a incorporação de evidências na saúde e na enfermagem. Texto Contexto Enferm 2008; 17(4):758-764.

13. Affleck W, Carmichael V, Whitley R. Men's Mental Health: Social Determinants and Implications for Services. Can J Psychiatry 2018; 63(9):581-589.

14. Lima M, Soares BGO, Mari JJ. Saúde e doença mental em Pelotas, RS: dados de um estudo populacional. Rev Psiquiatr Clín 1999; 26(5):225-235.

15. Schraiber LB, Figueiredo WS, Gomes R, Couto MT, Pinheiro TF, Machin R, Silva GSN, Valença O. Necessidades de saúde e masculinidades: atenção primária no cuidado aos homens. Cad Saude Publica 2010; 26(5):961-970.

16. Ludermir AB. Desigualdades de classe e gênero e saúde mental nas cidades. Physis 2008; 18(3):451-467.

17. Lipsky S, Caetano R, Roy-Byrne P. Triple jeopardy: impact of partner violence perpetration, mental health and substance use on perceived unmet need for mental health care among men. Soc Psychiatry Psychiatr Epidemiol 2011; 46(9):843-852.

18. Fleming PJ, Patterson TL, Chavarin CV, Semple SJ, Magis-Rodriguez C, Pitpitan EV. Are men's misogynistic attitudes associated with poor mental health and substance use behaviors? An exploratory study of men in Tijuana, Mexico. Psychol Men Masc 2018; 19(2):314-318 
19. Boettcher N, Mitchell J, Lashewicz B, Jones E, Wang J, Gundu S, Marchand A, Michalak E, Lam R. Men's Work-Related Stress and Mental Health: Illustrating the Workings of Masculine Role Norms. Am J Mens Health 2019; 13(2):1557988319838416.

20. Milner A, Shields M, King T. The Influence of Masculine Norms and Mental Health on Health Literacy Among Men: Evidence from the Ten to Men Study. Am J Mens Health 2019; 13(5):1557988319873532.

21. Whitley R. Men's Mental Health: Beyond Victim-Blaming. Can J Psychiatry 2018; 63(9):577-580.

22. Wong YJ, Owen J, Shea M. A latent class regression analysis of men's conformity to masculine norms and psychological distress. J Couns Psychol 2012; 59(1):176-183.

23. McKenzie SK, Collings S, Jenkin G, River J. Masculinity, Social Connectedness, and Mental Health: Men's Diverse Patterns of Practice. Am J Mens Health 2018; 12(5):1247-1261.

24. Keohane A, Richardson N. Negotiating Gender Norms to Support Men in Psychological Distress. Am J Mens Health 2018; 12(1):160-171.

25. Shafer K, Wendt D. Men's mental health: a call to social workers. Soc Work 2015; 60(2):105-112.

26. Buffel V, Van de Velde S, Bracke P. Professional care seeking for mental health problems among women and men in Europe: the role of socioeconomic, family -related and mental health status factors in explaining gender differences. Soc Psychiatry Psychiatr Epidemiol 2014; 49(10):1641-1653.

27. Parent MC, Hammer JH, Bradstreet TC, Schwartz EN, Jobe T. Men's Mental Health Help-Seeking Behaviors: An Intersectional Analysis. Am J Mens Health 2018; 12(1):64-73.

28. Cunha RB, Rebello LEFS, Gomes R. Como nossos pais? Gerações, sexualidade masculina e autocuidado. Physis 2012; 22(4):1419-1437.

29. Figueiredo W. Assistência à saúde dos homens: um desafio para os serviços de atenção primária. Cien Saude Colet 2005; 10(1):105-109.

30. Seidler ZE, Rice SM, Dhillon HM, Herrman H. Why it's time to focus on masculinity in mental health training and clinical practice. Australas Psychiatry 2019; 27(2):157-159.

31. Parent MC, Hammer JH, Bradstreet TC, Schwartz EN, Jobe T. Men's Mental Health Help-Seeking Behaviors: An Intersectional Analysis. Am J Mens Health 2018; 12(1):64-73.

32. Roy P, Tremblay G, Duplessis-Brochu É. Problematizing Men's Suicide, Mental Health, and Well-Being. Crisis 2018; 39(2):137-143.
33. Patrick S, Robertson S. Mental health and wellbeing: focus on men's health. Br J Nurs 2016; 25(21):11631169.

34. Cheshire A, Peters D, Ridge D. How do we improve men's mental health via primary care? An evaluation of the Atlas Men's Well-being Pilot Programme for stressed/distressed men. BMC Fam Pract 2016; 17:13.

35. Robertson S, Gough B, Hanna E, Raine G, Robinson M, Seims A, White A. Successful mental health promotion with men: the evidence from 'tacit knowledge'. Health Promot Int 2018; 33(2):334-344.

36. Ogrodniczuk J, Oliffe J, Kuhl D, Gross PA. Men's mental health: Spaces and places that work for men. Can Fam Physician 2016; 62(6):463-464.

37. Such E, Burton H, Copeland RJ, Davies R, Goyder E, Jeanes R, Kesterton S, Mackenzie K, Magee J. Developing a theory-driven framework for a football intervention for men with severe, moderate or enduring mental health problems: a participatory realist synthesis. J Ment Health 2020; 29(3):277-288.

38. Seidler ZE, Rice SM, Dhillon HM. Get angry or get even: Finding balance in the discussion of masculinity and mental health. Aust N Z J Psychiatry 2019; 53(11):1122.

39. Seaton CL, Bottorff JL, Jones-Bricker M, Oliffe JL, DeLeenheer D, Medhurst K. Men's Mental Health Promotion Interventions: A Scoping Review. Am J Mens Health 2017; 11(6):1823-1837.

40. Cassel EJ. The nature of suffering and goals of medicine. New York: Oxford University Press; 1991.

Artigo apresentado em 20/11/2020

Aprovado em 18/05/2021

Versão final apresentada em 20/05/2021

Editores-chefes: Romeu Gomes, Antônio Augusto Moura da Silva 